\title{
Magnetic Nanoparticle Based Nonviral MicroRNA Delivery into Freshly Isolated CD105 ${ }^{+}$hMSCs
}

\author{
Anna Schade, Paula Müller, Evgenya Delyagina, Natalia Voronina, Anna Skorska, \\ Cornelia Lux, Gustav Steinhoff, and Robert David
}

Department of Cardiac Surgery, Reference and Translation Center for Cardiac Stem Cell Therapy (RTC), University of Rostock, Schillingallee 35, 18057 Rostock, Germany

Correspondence should be addressed to Robert David; davidrob@med.uni-rostock.de

Received 31 January 2014; Accepted 2 March 2014; Published 31 March 2014

Academic Editor: Katherine Athayde Teixeira de Carvalho

Copyright (c) 2014 Anna Schade et al. This is an open access article distributed under the Creative Commons Attribution License, which permits unrestricted use, distribution, and reproduction in any medium, provided the original work is properly cited.

Genetic modifications of bone marrow derived human mesenchymal stem cells (hMSCs) using microRNAs (miRs) may be used to improve their therapeutic potential and enable innovative strategies in tissue regeneration. However, most of the studies use cultured hMSCs, although these can lose their stem cell characteristics during expansion. Therefore, we aimed to develop a nonviral miR carrier based on polyethylenimine (PEI) bound to magnetic nanoparticles (MNPs) for efficient miR delivery in freshly isolated hMSCs. MNP based transfection is preferable for genetic modifications in vivo due to improved selectivity, safety of delivery, and reduced side effects. Thus, in this study different miR/PEI and miR/PEI/MNP complex formulations were tested in vitro for uptake efficiency and cytotoxicity with respect to the influence of an external magnetic field. Afterwards, optimized magnetic complexes were selected and compared to commercially available magnetic vectors (Magnetofectamine, CombiMag). We found that all tested transfection reagents had high miR uptake rates (yielded over 60\%) and no significant cytotoxic effects. Our work may become crucial for virus-free introduction of therapeutic miRs as well as other nucleic acids in vivo. Moreover, in the field of targeted stem cell therapy nucleic acid delivery prior to transplantation may allowfor initial cell modulation in vitro.

\section{Introduction}

Bone marrow derived human mesenchymal stem cells (hMSCs) have been shown to bear great potential for cell based therapeutic strategies. The ability of these cells to differentiate into various cell types and to secrete a large spectrum of antiapoptotic, angiogenic, and immunomodulatory factors offers the possibility to use them for tissue repair [1-4]. Furthermore, hMSCs are characterized by the expression of specific stem cell surface markers (e.g., CD29, CD44, CD73, and CD105) and the absence of hematopoietic markers (e.g., CD45, CD117) [1]. Moreover, it was shown that CD105 (endoglin) is a suitable surface marker for efficient purification of hMSCs from bone marrow [2]. Currently, several clinical trials, which involve hMSCs for the treatment of graftversus-host disease, cartilage and meniscus repair, stroke, spinal cord injury, and Crohn's disease, are in progress [3]. Moreover, it has been recently shown that microRNA (miR) based genetic modifications of hMSCs before transplantation can significantly improve their therapeutic potential and survival rates [4-6]. Furthermore, miRs play an important role in stem cell regulation by influencing cell proliferation, differentiation, survival, apoptosis, and production of paracrine factors of hMSCs $[4,7,8]$. To date, several synthetic miRs are commercially available. However, miR delivery methods suitable for clinical applications will be crucial. Initially, viral carriers were widely used to transfer genetic material into target cells as they provide high transduction efficiency and long term gene expression. However, clinical applications of virus based gene carriers are limited as they may induce toxicity, immunogenicity, mutagenesis, and carcinogenesis $[9,10]$. Thus, various nonviral methods were developed. Nonviral vectors have the benefit to be noninflammatory, noninfectious, and less toxic for efficient delivery of nucleic acids [9]. To date, numerous nonviral transfection carriers based on cationic lipids and cationic polymers are available 
on the market (lipoplexes and polyplexes, resp.). Thereof, Lipofectamine and polyethylenimine (PEI) are the best investigated nonviral transfection reagents for efficient nucleic acid transfer [11-13]. However, the clinical applications of Lipofectamine and PEI are restricted due to sensitivity and safety issues. Moreover, nonviral carriers can be combined with magnetic nanoparticles in order to improve selectivity and safety of delivery as well as to decrease side effects [14]. In 2002, Scherer and coworkers invented a novel technique termed "magnetofection." This technique combines different well investigated gene delivery vectors (e.g., retrovirus, Lipofectamine, and PEI) with superparamagnetic iron oxide nanoparticles via salt-induced aggregation. The group showed that an externally applied magnetic field enhanced sedimentation of transfection complexes, thus improving transfection efficiency in vitro and in vivo [15]. In the last years, magnet based transfection (e.g., Magnetofectamine) has become a powerful tool for highly efficient and fast delivery of DNA $[15,16]$ as well as siRNA [17-19]. Our own group has developed a paramagnetic nonviral vector composed of nucleic acids condensed by biotinylated PEI and bound to streptavidin-coated iron oxide magnetic nanoparticles (MNPs) via biotin-streptavidin interactions. These MNP containing complexes, carrying therapeutic DNA, could be targeted by an external magnetic field to the site of interest in vivo [20]. Recently, we demonstrated that transfection with DNA/PEI/MNP complexes had a significantly higher transfection efficiency in cultivated hMSCs compared to DNA/PEI complexes even without the application of a magnetic field. We concluded a more rapid and efficient release of DNA from magnetic complexes compared to PEI polyplexes [21]. In contrast to DNA/PEI complexes, MNP containing complexes did not enter the nucleus due to strong biotin-streptavidin connections but released the DNA in the perinuclear region [14]. We have recently transferred this approach to transfection of hMSCs with $\mathrm{miR}$, as the latter binds to its target mRNAs in the proximity of the nucleus. In vitro, we could demonstrate that miR/PEI/MNP complexes had a better long term silencing effect compared to mere miR/PEI polyplexes, which might be beneficial for clinical applications [22].

Although freshly isolated hMSCs are more relevant to clinical use, the quantity of these cells is too low to reach the desired effect $[23,24]$. Thus, for our studies, as well as for clinical trials, hMSCs so far were expanded in vitro $[21,22,25]$. However, in vitro expansion of primary hMSCs is a costly and time-consuming procedure. In addition, the cells likely also lose their differentiation potential [2] and dramatically decrease their homing ability [26]. Therefore, genetic modifications of freshly isolated cells may be crucial to overcome these barriers and enable their clinical applications without previous in vitro expansion despite their low numbers available.

In this study, we applied a magnetic nonviral carrier for efficient miR transfection in freshly isolated hMSCs and compared it to commercially available magnetic vectors (Magnetofectamine, CombiMag particles) regarding uptake efficiency and cytotoxicity. We demonstrate that our novel magnetic transfection system is not inferior to the latter with respect to miR delivery and cellular tolerability.

\section{Material and Methods}

2.1. Isolation of $\mathrm{CD} 105^{+}$hMSCs. $\mathrm{CD} 105^{+}$cells were freshly isolated from sternal bone marrow. The bone marrow aspirates were obtained from patients during coronary artery bypass grafting at the Cardiac Surgery Department of the University of Rostock as previously described [27]. All donors gave their written consent to use their bone marrow for research proposes according to the Declaration of Helsinki.

At first, mononuclear cells (MNCs) were isolated by density gradient centrifugation. Afterwards, the $\mathrm{CD} 105^{+}$cell fraction was magnetically isolated using MACS technique according to the manufacturers' instructions (Miltenyi Biotec $\mathrm{GmbH}$, Bergisch Gladbach, Germany). Briefly, $1 \times 10^{7} \mathrm{MNCs}$ were incubated with $20 \mu \mathrm{L}$ of CD105 MicroBeads (Miltenyi Biotec $\mathrm{GmbH}$ ) for 30 minutes at $4^{\circ} \mathrm{C}$. Next, suspension cells were washed with MACS buffer containing $2 \mathrm{mM}$ EDTA (Gibco, Carlsbad, CA, USA), 0.5\% bovine serum albumin (BSA, Sigma-Aldrich, St. Louis, MO, USA), and PBS. Subsequently, magnetically labeled cells were loaded onto a MS MACS column (Miltenyi Biotec $\mathrm{GmbH}$ ) and placed in a magnetic field of a MiniMACS separator (Miltenyi Biotec $\mathrm{GmbH}$ ). Afterwards, the positive $\mathrm{CD}_{105}{ }^{+}$cell fraction was suspended in Mesenchymal Stem Cell Growth Medium (MSCGM, Lonza, Walkersville, MD, USA) containing $100 \mathrm{U} / \mathrm{mL}$ penicillin (PAA, Coelbe, Germany) and $100 \mu \mathrm{g} / \mathrm{mL}$ streptomycin (PAA). Isolated $\mathrm{CD} 105^{+}$cells were immediately used in further in vitro experiments or expanded in MSCGM (Lonza) at $37^{\circ} \mathrm{C}$ and $5 \% \mathrm{CO}_{2}$.

2.2. Immunophenotyping of $C D 105^{+}$hMSCs. Cell surface markers of freshly isolated and cultured $\mathrm{CD}_{105}{ }^{+}$hMSCs were fluorescently labeled with anti-human antibodies CD29APC, CD44-PerCP-Cy5.5, CD45-V500, CD73-PE, CD117PE-Cy7 (BD Biosciences, Heidelberg, Germany), and CD105AlexaFluor488 (AbD Serotec, Kidlington, UK). Respective mouse isotype antibodies served as negative controls. $3 \times 10^{4}$ events were acquired using BD FACS LSRII flow cytometer (BD Biosciences) and analyzed with BD FACSDiva Software 6 (BD Biosciences).

\subsection{Functional Differentiation Assay of $\mathrm{CD}_{105^{+}}$hMSCs.} Differentiation capacity of hMSCs was performed using the Human Mesenchymal Stem Cell Function Identification Kit (R\&D Systems, Minneapolis, MN, USA) according to the manufacturers' protocol. After 20 days under differentiation conditions, fatty acid binding protein-4 (FABP-4) and osteocalcin for adipogenic and osteogenic differentiation were fluorescently labeled, respectively. Nuclei were counter stained with $4^{\prime}, 6$-diamidino-2-phenylindol (DAPI, Invitrogen, Carlsbad, CA, USA). Samples were analyzed using ELYRA PS.1 LSM 780 microscope (Carl Zeiss, Jena, Germany) and ZEN2011 software (Carl Zeiss, Göttingen, Germany). 
2.4. Preparation of Polyplex Based Transfection Complexes. For preparation of polyplex based transfection complexes (miR/PEI, miR/PEI/MNP, and miR/PEI/CombiMag complexes), Cy3 dye-Labeled Pre-miR Negative Control number 1 (Ambion, Austin, TX, USA) for monitoring uptake efficiency and cytotoxicity and Pre-miR miRNA Precursor Molecules Negative Control number 1 (Ambion) for testing complex formation were used. Branched polyethylenimine (MW = $25 \mathrm{kDa}$, Sigma-Aldrich) was biotinylated as described previously [22] and was stored in aliquots at $4.41 \mathrm{mM}$ amine concentration at $4^{\circ} \mathrm{C}$.

Initially, miR/PEI complexes with different molar ratios of PEI nitrogen and miR phosphate (N/P ratios) were prepared as previously described [28]. Briefly, miR and PEI were diluted in equal volumes of $5 \%$ glucose solution, mixed, and incubated for 30 minutes at room temperature.

In order to form miR/PEI/MNP complexes, Streptavidin Magnesphere Paramagnetic Particles (Promega, Madison, WI, USA) were sonicated and filtered using $450 \mathrm{~nm}$ MillixHV PVDF syringe driven filter (Millipore, Tullagreen, Ireland). MNP filtrate was stored in aliquots at $4^{\circ} \mathrm{C}$. Afterwards, $1 \mu \mathrm{g} / \mathrm{mL}$ or $2 \mu \mathrm{g} / \mathrm{mL}$ MNPs was added to miR/PEI complexes and incubated for 30 minutes at room temperature.

For miR/PEI/CombiMag complex formation, CombiMag reagent (OZ Biosciences, Marseille, France) was sonicated for 20 minutes. Afterwards, $0.025 \mu \mathrm{L}$ CombiMag per 1 pmol miR $(0.025 \mu \mathrm{L}$ CombiMag/pmol) was mixed with miR/PEI complexes and incubated for 20 minutes at room temperature. All transfection complexes were freshly prepared before use.

2.5. Preparation of Lipoplex Based Transfection Complexes. For the formation of lipoplex based transfection complexes (miR/Magnetofectamine complexes), Cy3 dye-Labeled PremiR Negative Control number 1 (Ambion) was used for monitoring uptake efficiency and cytotoxicity.

At first, miR/Lipofectamine 2000 complexes were prepared. Therefore, miR and Lipofectamine 2000 transfection reagent ( $0.05 \mu \mathrm{L}$ Lipofectamine $2000 / \mathrm{pmol} \mathrm{miR}$, Invitrogen) were diluted separately each in $25 \mu \mathrm{L}$ of Opti-MEM I Reduced Serum Medium (Gibco) for 5 minutes at room temperature. Subsequently, miR and Lipofectamine 2000 solutions were mixed and incubated for 20 minutes at room temperature. For the formation of miR/Magnetofectamine complexes, CombiMag reagent (OZ Biosciences) was sonicated for 20 minutes. Afterwards, $0.025 \mu \mathrm{L}$ CombiMag/pmol was mixed with $\mathrm{miR} /$ Lipofectamine 2000 complexes as described above. Complexes were incubated for 20 minutes at room temperature according to Magnetofectamine instructions (OZ Biosciences). All transfection complexes were freshly prepared before use.

2.6. Condensation Assay of Transfection Complexes. The condensation of miR by PEI was studied by gel electrophoresis. $\mathrm{miR} / \mathrm{PEI}$ complexes were prepared as described above, mixed with loading dye (Fermentas GmbH, St. Leon-Rot, Germany), and loaded onto $2 \%$ agarose gel containing ethidium bromide. An electric field of $100 \mathrm{~V}$ was applied for 30 minutes and image was taken using TS imaging system (Biometra GmbH, Göttingen, Germany).

2.7. Transfection. For transfection experiments, $1 \times 10^{5}$ freshly isolated hMSCs per well were seeded in 48 well plates. Transfection complexes were prepared as described above and added drop by drop to the cells. Afterwards, cells were treated with and without the application of a magnetic field for 20 minutes using a Super Magnetic Plate (OZ Biosciences). Subsequently, cells were incubated for 24 hours at $37^{\circ} \mathrm{C}$ and $5 \% \mathrm{CO}_{2}$.

2.8. Evaluation of Uptake Efficiency and Cytotoxicity. For quantification of uptake efficiency, hMSCs were transfected with complexes containing Cy3 dye-Labeled Pre-miR Negative Control number 1 (Ambion) as described above for 24 hours. To investigate cytotoxicity, cells were stained with Near-IR LIVE/DEAD Fixable Dead Cell Stain Kit (Molecular Probes, Eugene, OR, USA). Moreover, cells were labeled with Alexa Fluor 488 mouse anti-human CD105 (clone SN6, AbD Serotec) and fixed with $4 \%$ PFA. $3 \times 10^{4}$ events were acquired using BD FACS LSRII flow cytometer (BD Biosciences) and analyzed with BD FACSDiva Software 6 (BD Biosciences). For determination of uptake efficiency, the number of living $\mathrm{CD} 105^{+} \mathrm{Cy} 3$-stained $\left(\mathrm{Cy}^{+}\right)$cells in relation to the total cell number of living $\mathrm{CD}_{105^{+}}$cells was measured. To evaluate complex cytotoxicity, the percentage of dead $\mathrm{CD}_{105}{ }^{+}$cells in relation to the total cell number of $\mathrm{CD} 105^{+}$cells was recorded.

2.9. Statistical Analysis. For all experiments, statistical analyses were performed by Student's $t$-test using SigmaPlot 11.0 software (Systat Software GmbH, Erkrath, Germany). Relative expression data of CD marker expressions are shown as mean \pm standard deviation (SD). All other values are presented as mean \pm standard error of the mean (SEM). A $P$ value $<0.05$ was considered to be statistically significant.

\section{Results and Discussion}

\subsection{Results}

3.1.1. Characterization of $\mathrm{CD} 105^{+}$hMSCs. $\mathrm{CD} 105^{+}$hMSCs were isolated from bone marrow and immediately characterized by multilineage differentiation and specific surface marker expression before use in further experiments [29, 30]. To investigate the differentiation capacity of hMSCs, cells were cultivated in adipogenic and osteogenic differentiation medium, respectively, and examined by fluorescent microscopy. Figure 1 illustrates that cells were able to differentiate into both adipocytes (Figure 1(a)) and osteocytes (Figure 1(b)). FACS analyses of freshly isolated $\mathrm{CD}_{105^{+}}$cells showed high expression of CD44 and CD105, a moderate expression of CD29 and CD45, and no expression of CD73 and CD117 (Figures 1(c) and 1(d)). Moreover, we compared the immunophenotype of freshly isolated cells with expanded $\mathrm{CD}_{105}{ }^{+}$cells. Cultivated $\mathrm{CD} 105^{+}$hMSCs presented high expressions of stem cell markers CD29, CD44, CD73, 
and CD105 but had downregulated expression of hematopoietic markers CD45 and CD117 (Figure 1(d)).

3.1.2. Characterization of Transfection Complexes. In order to examine condensation of miR by PEI, gel electrophoresis was performed. Thereby, different miR/PEI complexes with increasing amounts of PEI were investigated. Results demonstrated a big and sharp band for uncondensed miR that was used as a control (Figure 2(a)). At N/P ratios of 0.1, 0.25, and $0.5, \mathrm{miR}$ has partly formed complexes with PEI. Due to the bigger size, miR/PEI complexes remained in the slots, while uncondensed miR migrated through the gel. However, the miR signal disappeared completely at N/P ratios greater than 1 .

3.1.3. Transfection Optimization of miR/PEI Complexes. In order to optimize transfection of freshly isolated $\mathrm{CD} 105^{+}$ cells, polyplexes with different N/P ratios (N/P 2.5, N/P 10, and $\mathrm{N} / \mathrm{P} 33)$ and $\mathrm{miR}$ amounts (5 pmol and $10 \mathrm{pmol}$ ) were tested using flow cytometry. Complexes with an N/P ratio of 10 combined with $5 \mathrm{pmol} \mathrm{miR} \mathrm{(56 \% )} \mathrm{and} \mathrm{an} \mathrm{N/P} \mathrm{ratio} \mathrm{of}$ 2.5 combined with $10 \mathrm{pmol} \mathrm{miR}(69 \%)$ showed the highest uptake efficiencies (Figure 2(d)). In order to increase uptake rates, higher N/P ratios (N/P 33) were investigated. However, an $\mathrm{N} / \mathrm{P}$ ratio of 33 did not lead to further enhancement of uptake efficiencies. Likewise, potential cytotoxicity of the transfection complexes was investigated (Figure 2(e)). Thereby, untransfected cells were used as internal control (29\% dead cells) which is reflecting cytotoxicity of the isolation procedure. Transfection complexes with an N/P ratio of 10 combined with $5 \mathrm{pmol} \mathrm{miR}$ led to moderately increased cell mortality (17\%) compared to controls. Yet, polyplexes with an N/P ratio of 2.5 combined with $10 \mathrm{pmol}$ miR showed no significant differences compared to controls (32\% versus $29 \%$ ). However, an increase in the N/P ratio (N/P 33) did lead to higher cytotoxicity in polyplexes with $5 \mathrm{pmol}$ $\mathrm{miR}(57 \%)$ as well as with $10 \mathrm{pmol} \mathrm{miR} \mathrm{(55 \% ).} \mathrm{Therefore,} \mathrm{with}$ respect to the highest uptake rates and lowest cytotoxicity, polyplexes composed of an N/P ratio of 10 combined with $5 \mathrm{pmol} \mathrm{miR}$ and complexes with an N/P ratio of 2.5 combined with $10 \mathrm{pmol} \mathrm{miR}$ were considered to represent optimal compositions and were utilized in further experiments.

\subsubsection{Transfection Optimization of miR/PEI/MNP Complexes.}

In order to increase selectivity of the carrier and safety for clinical applications, the two optimized miR/PEI complexes were combined with different MNP amounts ( 1 or $2 \mu \mathrm{g} / \mathrm{mL}$ MNPs). Therefore uptake rates (Figures 3(a) and 3(c)) and cytotoxicity (Figures $3(\mathrm{~b})$ and $3(\mathrm{~d})$ ) of the different complex compositions as well as the influence of a magnetic field were investigated by flow cytometry. Magnetic polyplexes consisting of an N/P ratio of 10 combined with 5 pmol $\mathrm{miR}$ had similar uptake rates (up to $76 \%$ ) compared to the corresponding miR/PEI complexes (81\%) without the application of a magnetic field (Figure 3(a)). Moreover, cytotoxicity of these complexes was investigated. Magnetic polyplexes with 1 or $2 \mu \mathrm{g} / \mathrm{mL}$ MNPs (27\% versus $24 \%$ ) showed no cytotoxic effect when compared to miR/PEI complexes
(30\%, Figure 3(b)). Likewise, polyplexes consisting of an N/P ratio of 2.5 combined with $10 \mathrm{pmol}$ miR coupled to MNPs were investigated. Uptake rates of magnetic complexes with 1 or $2 \mu \mathrm{g} / \mathrm{mL}$ MNPs ( $\sim 65 \%)$, respectively, did not significantly differ compared to appropriate miR/PEI complexes (77\%) in the absence of a magnetic field (Figure 3(c)). Furthermore, no significant differences in cytotoxicity were observed between the transfected cells (ranging from $15 \%$ to $18 \%$ ) and the control (17\%, Figure 3(d)). Additionally, the influence of a magnetic field was investigated. However, no significant improvement of uptake efficiency and reduction of cytotoxicity were observed when an external magnetic field was applied (Figure 3). For further experiments, two magnetic complexes were used: first an N/P ratio of 10 combined with $5 \mathrm{pmol} \mathrm{miR}$ bound to $1 \mu \mathrm{g} / \mathrm{mL}$ MNPs and second an N/P ratio of 2.5 combined with $10 \mathrm{pmol} \mathrm{miR}$ bound to $1 \mu \mathrm{g} / \mathrm{mL}$ MNPs.

3.1.5. Comparison of miR/PEI/MNP Complexes to Established Transfection Reagents. Optimized magnetic polyplexes were compared to commercially available magnetic vectors: Magnetofectamine and CombiMag particles. Therefore uptake efficiency (Figures 4(a) and 4(c)) and cytotoxicity (Figures 4(b) and 4(d)) of different transfection complexes were investigated using flow cytometry. miR/PEI/MNP complexes with an N/P ratio of 10 combined with 5 pmol miR bound to $1 \mu \mathrm{g} / \mathrm{mL}$ MNPs showed high uptake rates $(68 \%$, Figure 4(a)). Moreover, no significant differences in uptake efficiency were observed between miR/PEI/CombiMag (64\%) and $\mathrm{miR} /$ Magnetofectamine (59\%) complexes. Mortality between treated and control cells was comparable and ranged between $11 \%$ and $17 \%$ (Figure $4(\mathrm{~b})$ ). Moreover, uptake efficiency and cytotoxicity of miR/PEI/MNP complexes composed of an N/P ratio of 2.5 combined with $10 \mathrm{pmol} \mathrm{miR}$ bound to $1 \mu \mathrm{g} / \mathrm{mL}$ MNPs were investigated and compared to known transfection reagents. Uptake rates of $\mathrm{miR} / \mathrm{PEI} / \mathrm{MNP}$ complexes reached up to $79 \%$ and were compared to $\mathrm{miR} / \mathrm{PEI} / \mathrm{CombiMag}(56 \%)$ and miR/Magnetofectamine (75\%, Figure 4(c)). Moreover, cell mortality of transfected cells (ranging from 9\% to 14\%) was not significantly different when compared to untreated control (9\%, Figure 4(d)).

3.2. Discussion. Therapeutic potential of hMSCs can be improved by genetic modifications with distinct miRs [5, 6]. Our group has recently developed a magnetic nonviral transfection carrier consisting of biotinylated PEI bound to streptavidin-coated MNPs for highly efficient miR delivery $(\sim 75 \%)$ and applied it to cultivated hMSCs [22]. In general, hMSCs can be characterized by multilineage differentiation potential and the expression of specific surface markers (e.g., CD29, CD44, CD73, and CD105) [1]. Our previous studies have demonstrated that the purified $\mathrm{CD} 05^{+}$fraction of expanded hMSCs had a beneficial effect in cardiac tissue regeneration [27]. However, the time-consuming, expensive, and potentially detrimental cell expansion process might be avoidable as it has previously been described by Aslan et al. They tested $\mathrm{CD} 105^{+}$cells freshly isolated from bone marrow in vitro and in vivo. Importantly, these cells were indeed 


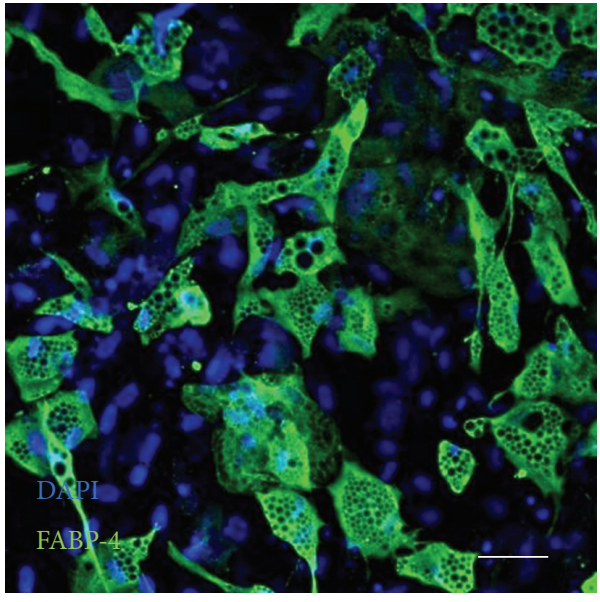

(a)
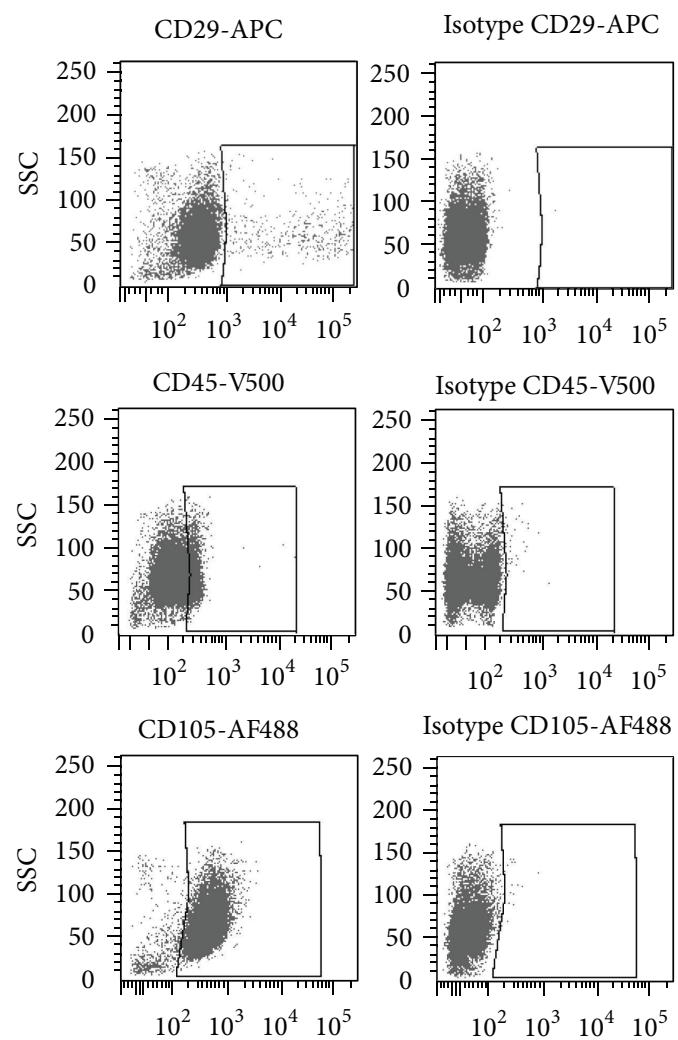

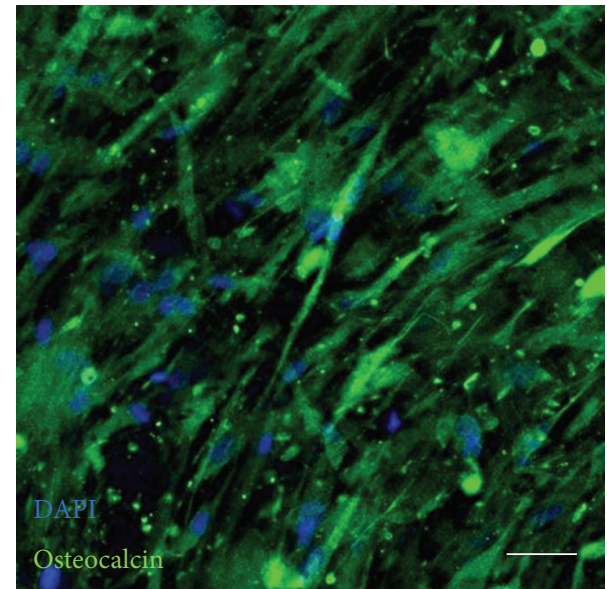

(b)

CD44-PerCP-Cy5.5 Isotype CD44-PerCP-Cy5.5
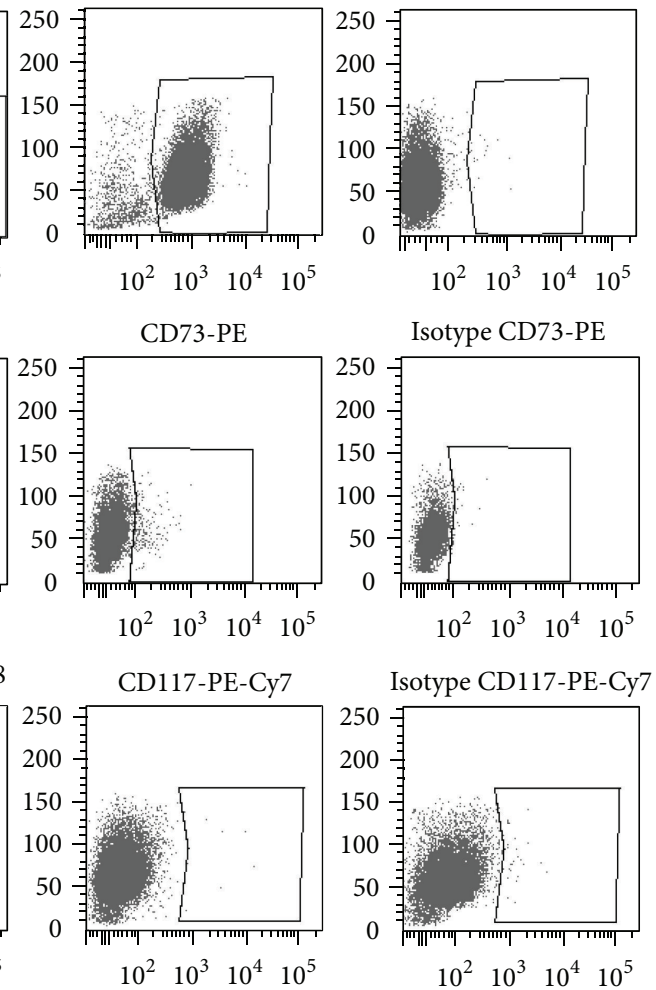

(c)

\begin{tabular}{l|cccccc}
\hline & CD29 & CD44 & CD45 & CD73 & CD105 & CD117 \\
\hline Fresh hMSCs & $4.8 \% \pm 2.4$ & $93.8 \% \pm 1.3$ & $23.8 \% \pm 0.6$ & $1.4 \% \pm 0.9$ & $93.0 \% \pm 4.6$ & $0.1 \% \pm 0.1$ \\
& & & & & & \\
Cultured hMSCs & $99.6 \% \pm 0.4$ & $99.7 \% \pm 0.3$ & $0.5 \% \pm 0.5$ & $97.5 \% \pm 3.3$ & $98.5 \% \pm 0.4$ & $0.2 \% \pm 0.2$ \\
\hline
\end{tabular}

(d)

Figure 1: Characterization of CD105 ${ }^{+}$hMSCs. ((a), (b)) Functional differentiation capacity of freshly isolated CD105 ${ }^{+}$cells was shown by immunostaining of FABP-4 (green) for adipocytes (a) and osteocalcin (green) for osteocytes (b) after 20 days in appropriate differentiation medium. Nuclei were counter stained with DAPI (blue). Scale bars $=50 \mu \mathrm{m}$. ((c), (d)) Immunophenotyping of freshly isolated ((c), (d)) and cultured $\mathrm{CD}_{105^{+}}$hMSCs (d) was evaluated by flow cytometry after staining of specific cell surface markers. Corresponding isotype controls were used as negative controls (c). The relative expression values of CD marker expression are shown as mean \pm SD; $n=2$ (d). 


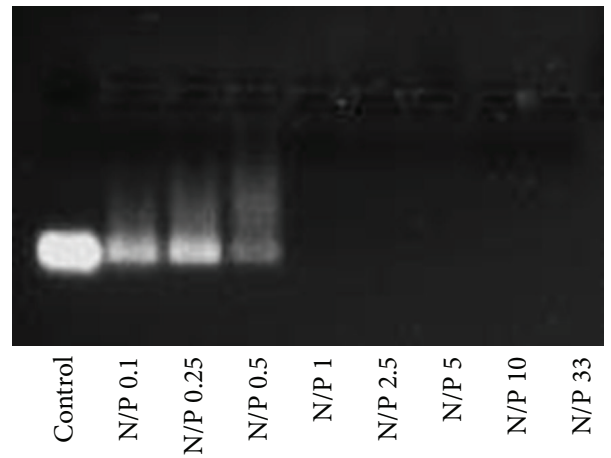

(a)

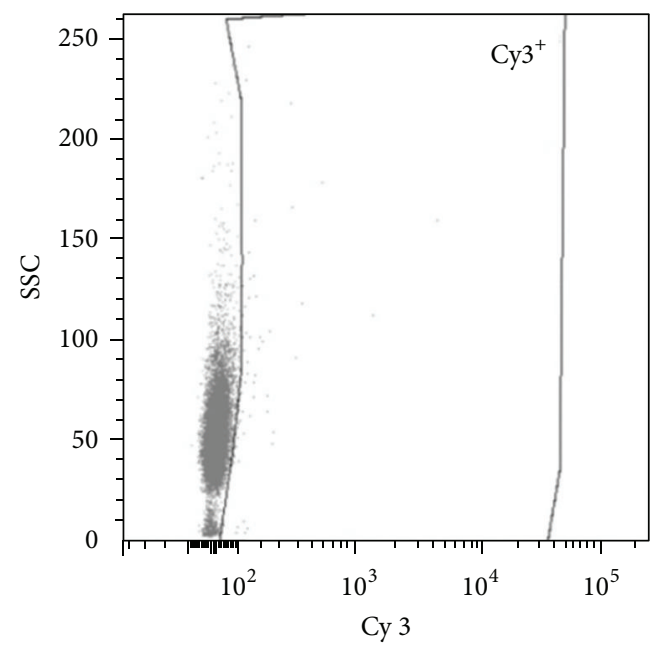

(b)

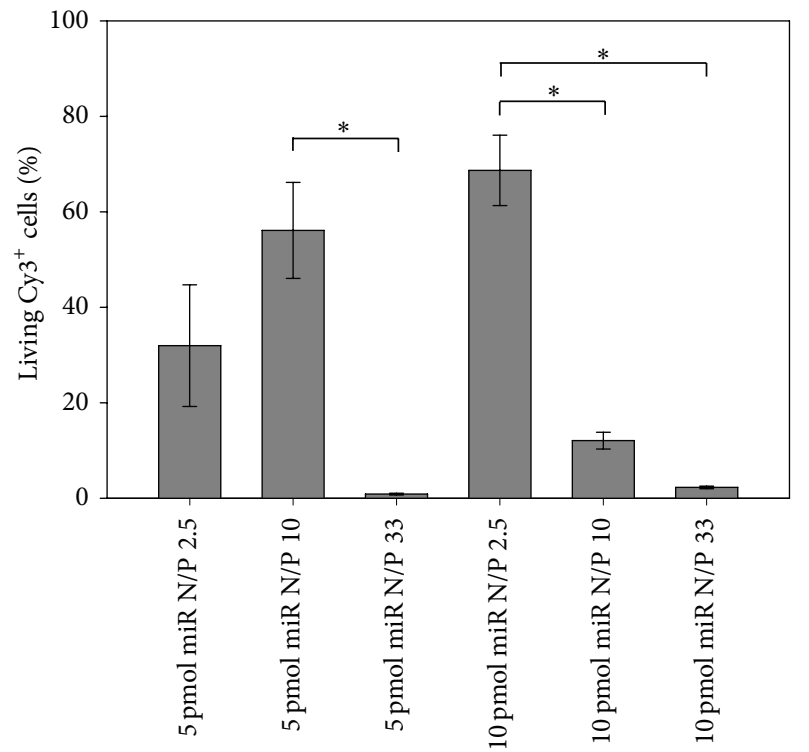

(d)

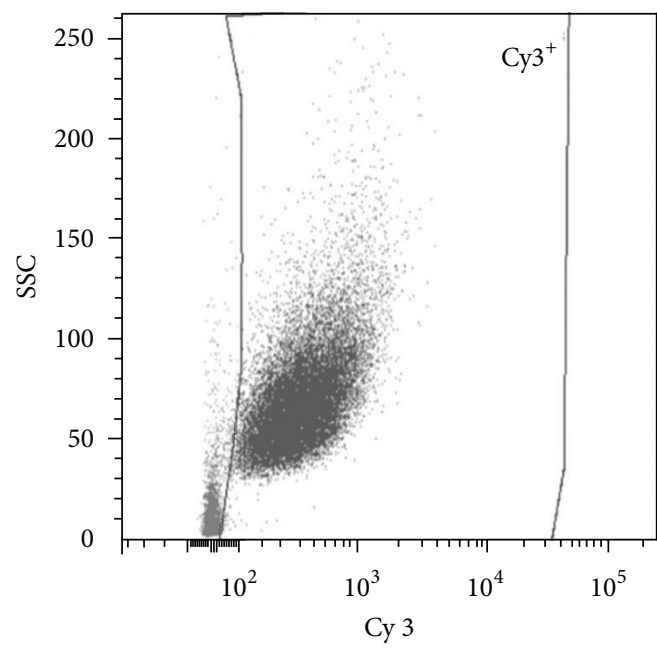

(c)

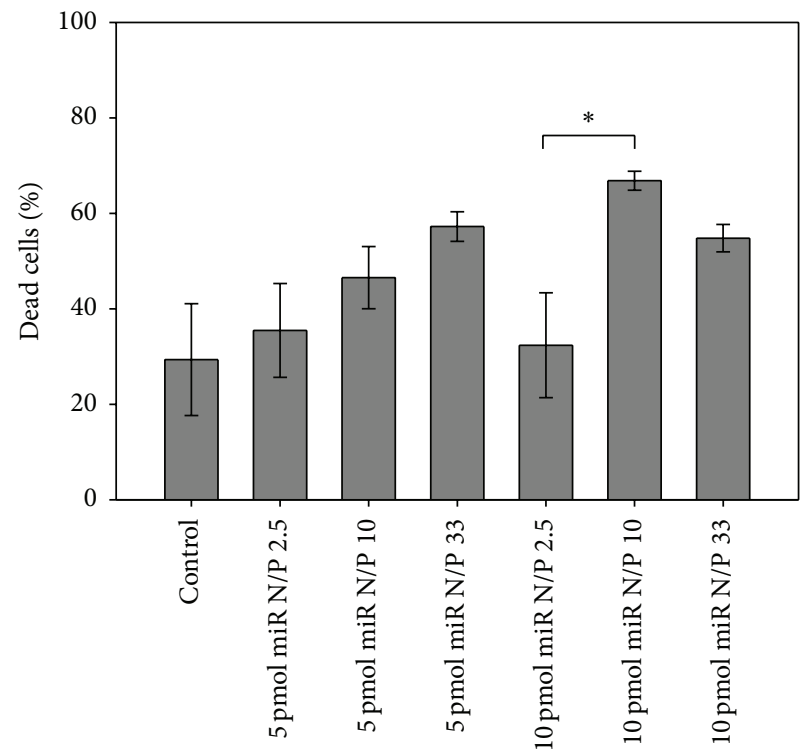

(e)

FIgURE 2: Characterization and transfection optimization of miR/PEI complexes. (a) Condensation of miR/PEI complexes composed of $20 \mathrm{pmol} \mathrm{miR}$ and different N/P ratios (ranging between 0.1 and 33) was analysed by gel electrophoresis. miR alone served as positive control. ((b), (c)) Gating strategy of FACS measurements. (b) Untransfected living CD105 $5^{+}$cells were used as negative control. (c) Living CD105 ${ }^{+}$cells transfected with Cy3-labeled complexes. ((d), (e)) Optimization of miR amounts and N/P ratios for transfection of freshly isolated hMSCs. hMSCs were transfected with Cy3-labeled miR/PEI complexes consisting of three different N/P ratios (N/P 2.5, N/P 10, and N/P 33) and two different miR amounts ( 5 and $10 \mathrm{pmol}$ ). 24 hours after transfection, uptake efficiency (d) and cytotoxicity (e) of complexes were analyzed by flow cytometry. Untransfected cells were used as control. Values are presented as mean $\pm \mathrm{SEM} ; n=3 ; *=P \leq 0.05$. 


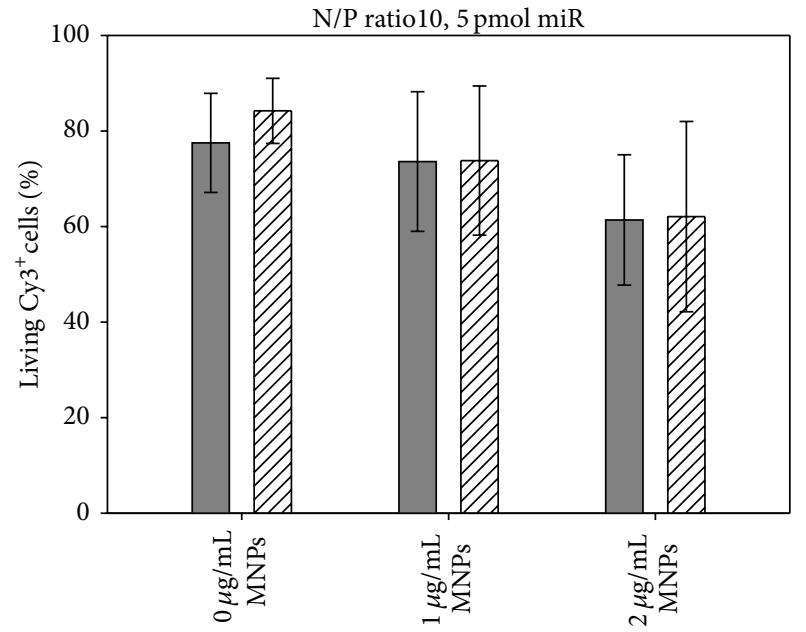

(a)

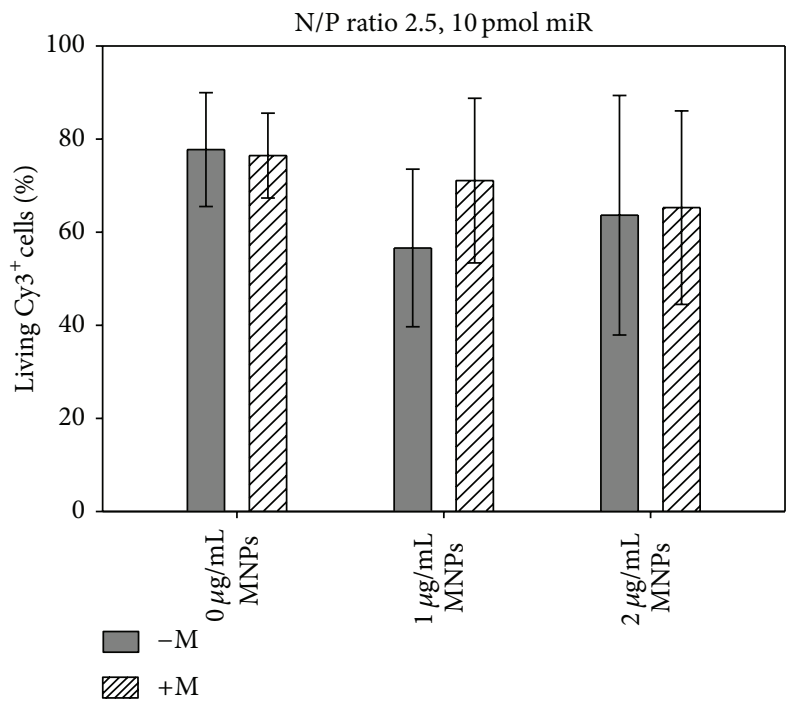

(c)

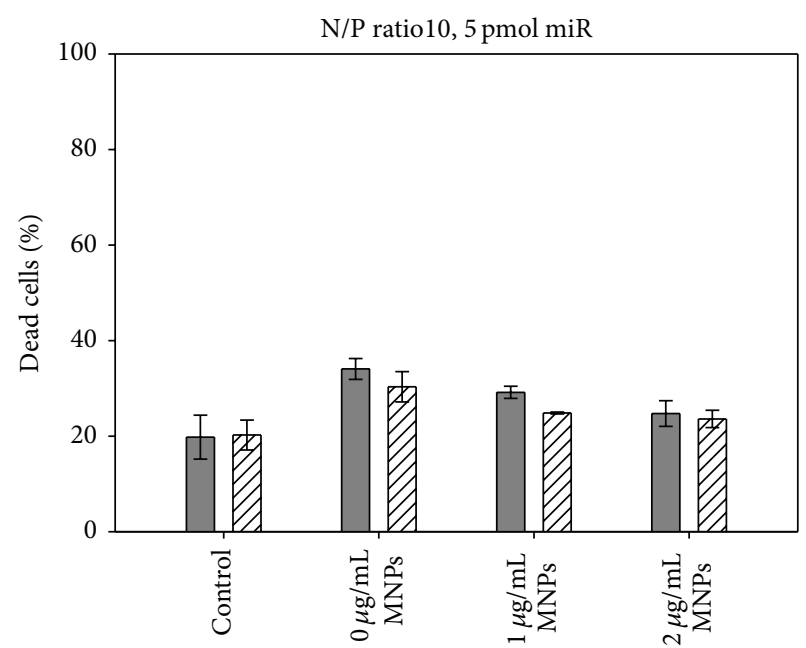

(b)

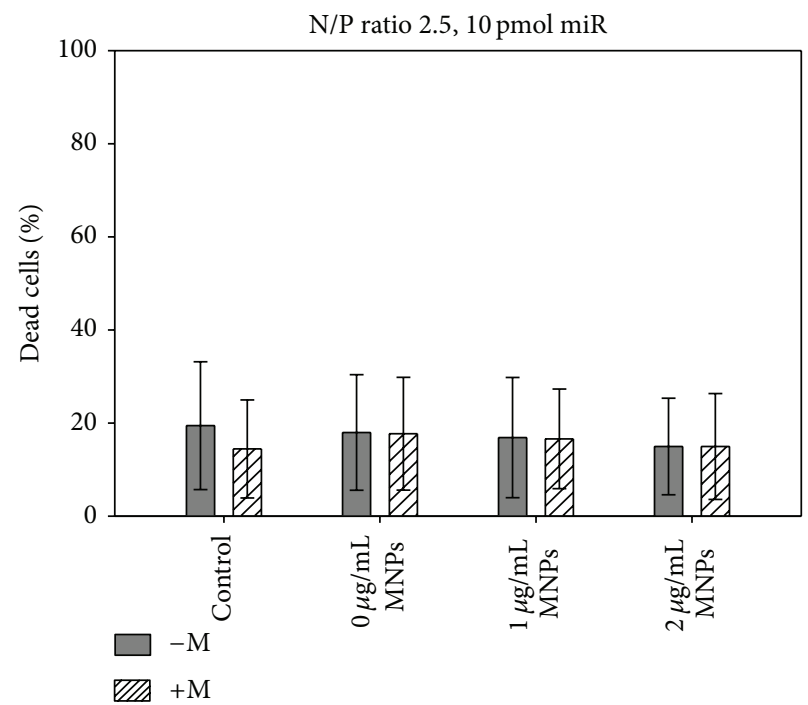

(d)

FIgURE 3: Transfection optimization of miR/PEI/MNP complexes. ((a)-(d)) Optimization of MNP amounts for transfection of freshly isolated hMSCs with $(+\mathrm{M})$ and without $(-\mathrm{M})$ the application of a magnetic field. hMSCs were transfected with Cy3-labeled miR/PEI and $\mathrm{miR} / \mathrm{PEI} / \mathrm{MNP}$ complexes composed of an N/P ratio of 10 combined with $5 \mathrm{pmol} \mathrm{miR}((\mathrm{a})$, (b)) or an N/P ratio of $2.5 \mathrm{combined}$ with $10 \mathrm{pmol} \mathrm{miR}((\mathrm{c}),(\mathrm{d}))$ and three different MNP amounts $(0,1$, or $2 \mu \mathrm{g} / \mathrm{mL}$ MNPs). 24 hours after transfection, uptake efficiency ((a), (c)) and cytotoxicity ((b), (d)) of complexes were analyzed by flow cytometry. Untransfected cells were used as control. Values are presented as mean \pm SEM; $n=3$.

capable of giving rise to hMSCs bearing all typical properties after expansion [2]. Likewise in our study, freshly isolated $\mathrm{CD} 105^{+}$cells showed a slightly altered immunophenotype when compared to hMSCs after expansion (Figures 1(c) and 1(d)). However, our freshly isolated $\mathrm{CD} 05^{+}$cells also did adhere to plastic surfaces and showed a morphology and phenotype typical for hMSCs in expansion culture (Figure 1(d)). Moreover, we proofed the ability of these hMSCs derived from our freshly isolated $\mathrm{CD} 105^{+}$cells to differentiate into adipocytes (Figure 1(a)) and osteocytes (Figure 1(b)) under appropriate culture conditions. This clearly confirmed their stem cell character. Therefore, our findings are in line with the report of Aslan et al. [2].

To optimize miR transfection of freshly isolated $\mathrm{CD} 105^{+}$ hMSCs, we initially tested miR/PEI complexes with different amounts of miR and PEI. In order to investigate the physicochemical properties of various miR/PEI complexes, condensation of miR by PEI was analyzed using gel electrophoresis (Figure 2(a)). Results demonstrated that, at N/P ratios from 1 to 33 , no signals were observed. Therefore, we concluded that an N/P ratio 1 was sufficient to condense the whole amount of miR that is in correspondence with our previous findings [22]. Appropriate condensation of miR is essential for effective delivery into cells as it is protecting $\mathrm{miR}$ from early enzymatic degradation [11]. Furthermore, it prevents the activation of the innate immune system by double stranded RNA [31].

In order to establish efficient transfection complexes for miR delivery in freshly isolated $\mathrm{CD} 105^{+} \mathrm{hMSCs}$, miR/PEI complexes with different $\mathrm{N} / \mathrm{P}$ ratios $(2.5,10$, and 33$)$ and $\mathrm{miR}$ 


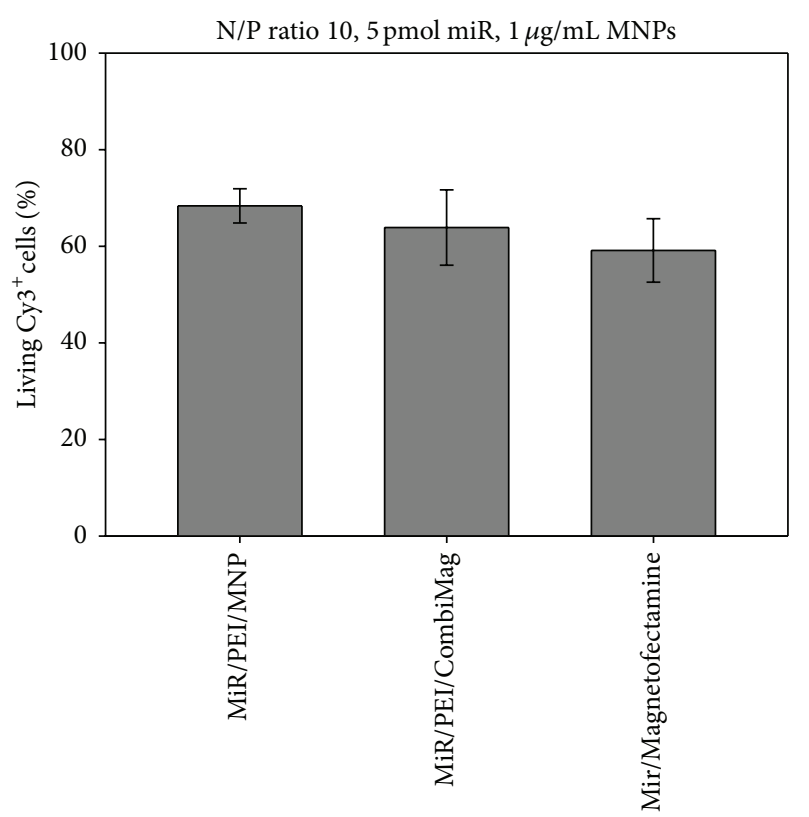

(a)

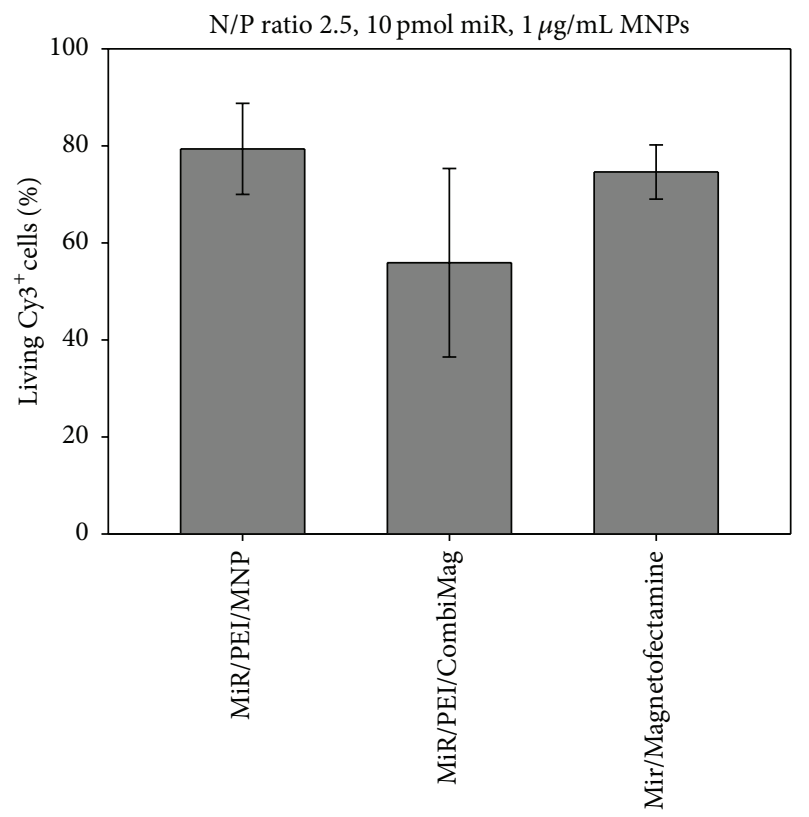

(c)

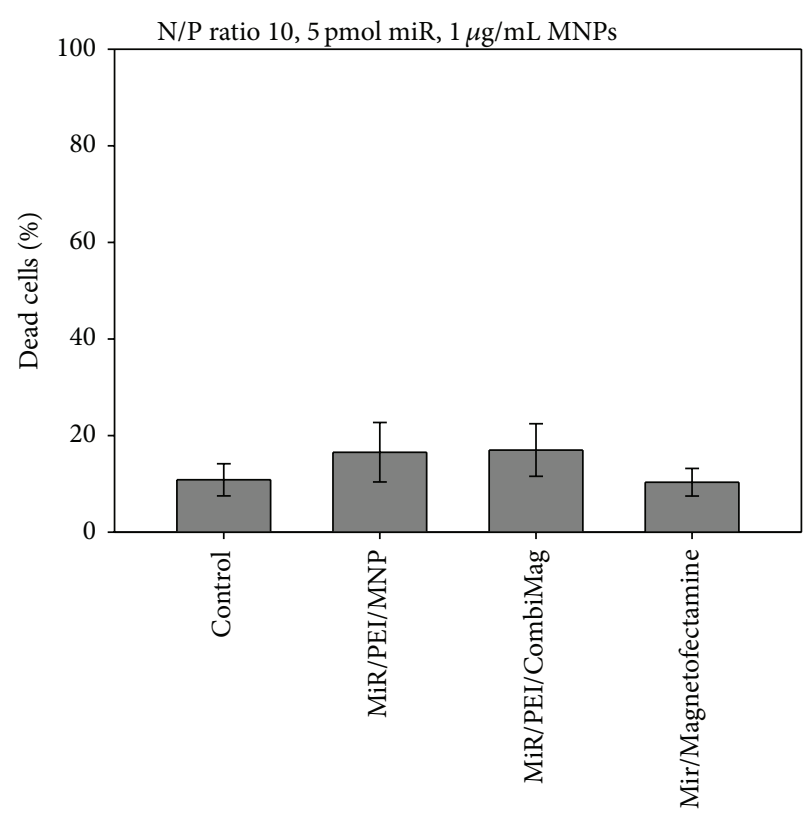

(b)

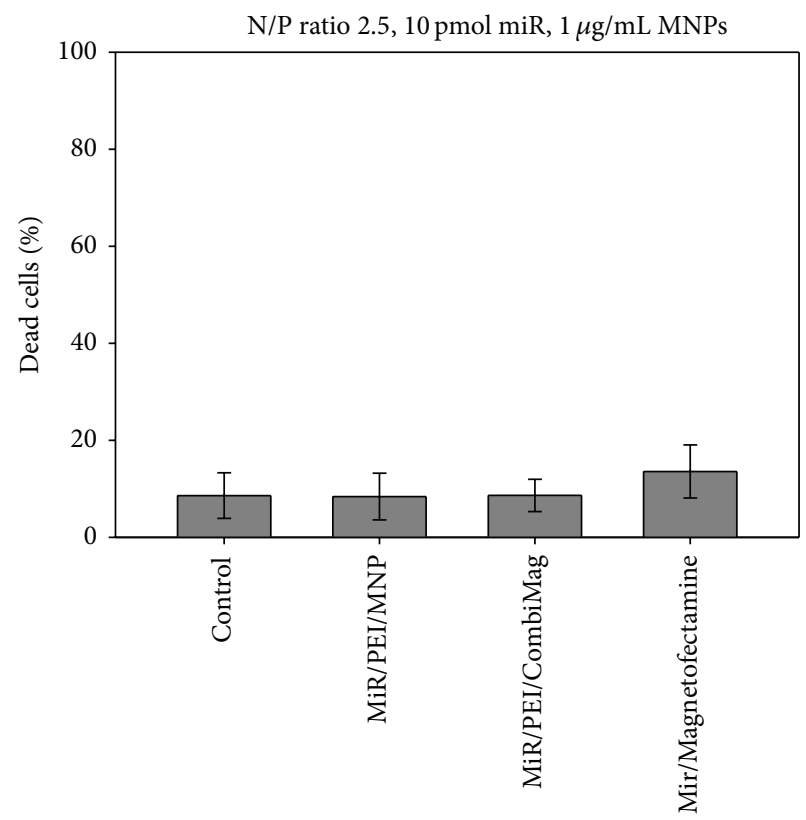

(d)

FIGURE 4: Comparison between optimized miR/PEI/MNP complexes and commercially available magnetic transfection reagents. Freshly isolated hMSCs were transfected with Cy3-labeled miR/PEI/MNP complexes composed of an N/P ratio of 10 combined with 5 pmol miR bound to $1 \mu \mathrm{g} / \mathrm{mL}$ MNPs ((a), (b)) or an N/P ratio of 2.5 combined with $10 \mathrm{pmol} \mathrm{miR} \mathrm{bound} \mathrm{to} 1 \mu \mathrm{g} / \mathrm{mL} \mathrm{MNPs}((\mathrm{c})$, (d)). For comparison with commercially available magnetic transfection complexes, appropriate amounts of Lipofectamine 2000 and CombiMag particles were used. 24 hours after transfection, uptake efficiency $((\mathrm{a}),(\mathrm{c}))$ and cytotoxicity $((\mathrm{b}),(\mathrm{d}))$ of complexes were determined by flow cytometry, while a magnetic field was applied. Untransfected cells were used as control. Values are presented as mean \pm SEM; $n=3$.

amounts (5 and $10 \mathrm{pmol}$ ) were tested (Figures 2(c) and 2(d)). Previous experiments showed that N/P ratios can significantly influence uptake efficiency of transfection complexes [32]; thus we selected 3 different N/P ratios for optimization experiments. Delyagina et al. showed that an N/P ratio of 2.5 showed the highest uptake efficiency of plasmid DNA in cultured hMSCs [21]. We have also demonstrated that miR was efficiently delivered using the same vector with N/P ratios of 10 and 33 [22]. Our current results demonstrated that miR/PEI complexes with 5 pmol provided the highest uptake efficiency at an N/P ratio of 10 (56\%) for fresh hMSCs. In contrast to miR transfection in expanded hMSCs, we could further enhance miR uptake in freshly isolated hMSCs by increasing the miR amount to $10 \mathrm{pmol}$ with an $\mathrm{N} / \mathrm{P}$ ratio of $2.5(69 \%)$. Due to the low N/P ratio, cytotoxicity could be decreased and was comparable to controls (32\% versus 
$29 \%)$. The strategy of using low N/P ratios reduced the amount of used PEI that might be advantageous for genetic modifications. That is especially important for freshly isolated hMSCs as they might react very sensitive to potentially toxic reagents. Therefore, we decided to use miR/PEI complexes with an $\mathrm{N} / \mathrm{P}$ ratio of 2.5 combined with $10 \mathrm{pmol} \mathrm{miR}$ for further experiments and compare it to complexes consisting of an N/P ratio of 10 combined with $5 \mathrm{pmol}$ miR which were used in our previous work [22].

Furthermore, we combined optimized miR/PEI complexes with different MNP amounts to enable magnetic targeting of transfected cells (Figure 3). Based on previous work, $\mathrm{miR} / \mathrm{PEI} / \mathrm{MNP}$ complexes with $1 \mu \mathrm{g} / \mathrm{mL}$ or $2 \mu \mathrm{g} / \mathrm{mL}$ MNPs were investigated as they showed a significant increase in uptake efficiency compared to control (75\% versus 50\%) [22]. Our current investigations showed no significant differences in uptake rates and cytotoxicity of magnetic complexes with different compositions. The values of uptake efficiencies were comparable to those previously obtained for cultivated hMSCs [22] and were in the range from 56\% to $79 \%$ (Figures 3(a) and 3(c)). Moreover, cell mortality was comparable to controls representing the basic level of cytotoxicity due to the isolation process (Figures 3(b) and $3(\mathrm{~d})$ ). Additionally, we investigated the influence of a magnetic field on miR transfection into freshly isolated cells. We observed no significant differences in uptake efficiency and cytotoxicity in cells transfected in the presence or absence of a magnetic field (Figure 3). These findings are in agreement with our previous data that showed efficient plasmid DNA transfection in hMSCs without the application of an external magnetic field [21]. This effect might be explained by the fact that a magnetic field has no influence on cellular uptake or intracellular transfection mechanism, as previously proposed [33].

Furthermore, we compared the performance of our $\mathrm{miR} / \mathrm{PEI} / \mathrm{MNP}$ vector and commercially available magnetic transfection reagents in freshly isolated $\mathrm{CD}_{105^{+}}$cells (Figure 4). Therefore we chose magnetic complexes consisting of an $\mathrm{N} / \mathrm{P}$ ratio of 10 combined with $5 \mathrm{pmol} \mathrm{miR}$ bound to $1 \mu \mathrm{g} / \mathrm{mL}$ MNPs as they showed good transfection values in both freshly isolated and cultured hMSCs [22]. Additionally, we selected complexes with an N/P ratio of 2.5 combined with $10 \mathrm{pmol} \mathrm{miR}$ bound to $1 \mu \mathrm{g} / \mathrm{mL}$ MNPs as lower amount of PEI and MNPs in their composition facilitates their application. Magnetofectamine is a combined transfection reagent which consists of Lipofectamine 2000 and CombiMag particles and can be used for delivery of different nucleic acids in various cell types in the presence of an external magnetic field [34]. Lipofectamine is the most effective and best investigated cationic lipid, which can interact spontaneously with nucleic acids through electrostatic interactions forming stable lipoplexes $[13,35]$. CombiMag particles can also serve as transfection reagent when combined with cationic polymers or cationic lipids [34]. However, the combination of CombiMag particles with Lipofectamine 2000 is proposed as the most effective approach. [34]. We could demonstrate that our $\mathrm{miR} / \mathrm{PEI} / \mathrm{MNP}$ vector was able to reach uptake rates similar to Magnetofectamine and $\mathrm{miR} / \mathrm{PEI} / \mathrm{CombiMag}$ complexes (Figures 4(a) and 4(c)). Moreover, cytotoxicity of all transfection complexes was comparable to controls indicating no cytotoxic effect of the different nonviral complexes on freshly isolated hMSCs (Figures 4(b) and 4(d)).

It has previously been shown by our group that both nonviral and viral magnetic complexes carrying labeled or therapeutic plasmid DNA can be targeted in vivo $[20,36]$. Yet, thereby, no transfection into stem cells was performed. Furthermore, miR as nucleic acid of interest has so far not been used for in vivo targeting. This shortcoming led us to transfer our approach to an efficient delivery of $\mathrm{miR}$ into freshly isolated $\mathrm{CD} 105^{+}$hMSCs using three different magnetic carrier systems for the first time. All systems investigated yielded around $65 \%$ uptake of labeled miR combined with high cell viability. Therefore, we here introduce a novel carrier system which provides equal efficiency and cellular tolerance as commercially available magnetic transfection reagents.

Overall, the specific properties of the respective magnetic carriers for various applications may allow for direct nonviral delivery of therapeutic miRs as well as other nucleic acids in vivo. Moreover, introduction of these nucleic acids in vitro prior to cell transplantation may enable stem cell modulation for targeted stem cell therapy.

\section{Conclusion}

In this report, we successfully developed magnetic nonviral carriers for efficient miR transfection into freshly isolated $\mathrm{CD}_{105}{ }^{+}$hMSCs. These magnetic vectors were able to reach high uptake rates (68\% versus $79 \%$ ) with no significant cytotoxic effect. The performance of the novel miR carrier equaled that of commercially available magnetic transfection reagents. Magnetic nanoparticle based miR transfection may become important to optimize stem cells meant for transplantation, although further preclinical experiments are required.

\section{Conflict of Interests}

The authors declare that there is no conflict of interests regarding the publication of this paper.

\section{Authors' Contribution}

Anna Schade and Paula Müller contributed equally to this work.

\section{Acknowledgments}

This work was supported by ESF Grant (UR11 041; ESF/IVBM-B35-0010/12); the Reference and Translation Center for Cardiac Stem Cell Therapy (BMBF: FKZ 0312138A); and Land MV (FKZ V630-F-075-2010/183; V630-S075-2010/185). Robert David is supported by the DFG (DA1296/2-1). 


\section{References}

[1] M. Dominici, K. le Blanc, I. Mueller et al., "Minimal criteria for defining multipotent mesenchymal stromal cells. The International Society for Cellular Therapy position statement," Cytotherapy, vol. 8, no. 4, pp. 315-317, 2006.

[2] H. Aslan, Y. Zilberman, L. Kandel et al., "Osteogenic differentiation of noncultured immunoisolated bone marrow-derived CD105 cells," Stem Cells, vol. 24, no. 7, pp. 1728-1737, 2006.

[3] A. I. Caplan, "Adult mesenchymal stem cells for tissue engineering versus regenerative medicine," Journal of Cellular Physiology, vol. 213, no. 2, pp. 341-347, 2007.

[4] Z. Wen, S. Zheng, C. Zhou, W. Yuan, J. Wang, and T. Wang, "Bone marrow mesenchymal stem cells for post-myocardial infarction cardiac repair: microRNAs as novel regulators," Journal of Cellular and Molecular Medicine, vol. 16, no. 4, pp. 657-671, 2012.

[5] J. J. Chen and S. H. Zhou, "Mesenchymal stem cells overexpressing MiR-126 enhance ischemic angiogenesis via the AKT/ERKrelated pathway," Cardiology Journal, vol. 18, no. 6, pp. 675-681, 2011.

[6] F. Huang, X. Zhu, X. Q. Hu et al., "Mesenchymal stem cells modified with miR-126 release angiogenic factors and activate Notch ligand Delta-like-4, enhancing ischemic angiogenesis and cell survival," International Journal of Molecular Medicine, vol. 31, no. 2, pp. 484-492, 2013.

[7] M. Tome, P. Lopez-Romero, C. Albo et al., "miR-335 orchestrates cell proliferation, migration and differentiation in human mesenchymal stem cells," Cell Death and Differentiation, vol. 18, no. 6, pp. 985-995, 2011.

[8] P. Jakob and U. Landmesser, "Role of microRNAs in stem/progenitor cells and cardiovascular repair," Cardiovascular Research, vol. 93, no. 4, pp. 614-622, 2012.

[9] H. Boulaiz, J. A. Marchal, J. Prados, C. Melguizo, and A. Aranega, "Non-viral and viral vectors for gene therapy," Cellular and Molecular Biology, vol. 51, no. 1, pp. 3-22, 2005.

[10] F. H. Lin, C. H. Chen, W. T. Cheng, and T. F. Kuo, "Modified montmorillonite as vector for gene delivery," Biomaterials, vol. 27, no. 17, pp. 3333-3338, 2006.

[11] T. Bieber, W. Meissner, S. Kostin, A. Niemann, and H. P. Elsasser, "Intracellular route and transcriptional competence of polyethylenimine-DNA complexes," Journal of Controlled Release, vol. 82, no. 2-3, pp. 441-454, 2002.

[12] M. A. Mintzer and E. E. Simanek, "Nonviral vectors for gene delivery," Chemical Reviews, vol. 109, no. 2, pp. 259-302, 2009.

[13] S. Zhang, B. Zhao, H. Jiang, B. Wang, and B. Ma, "Cationic lipids and polymers mediated vectors for delivery of siRNA," Journal of Controlled Release, vol. 123, no. 1, pp. 1-10, 2007.

[14] E. Delyagina, W. Li, N. Ma, and G. Steinhoff, "Magnetic targeting strategies in gene delivery," Nanomedicine, vol. 6, no. 9, pp. 1593-1604, 2011.

[15] F. Scherer, M. Anton, U. Schillinger et al., "Magnetofection: enhancing and targeting gene delivery by magnetic force in vitro and in vivo," Gene Therapy, vol. 9, no. 2, pp. 102-109, 2002.

[16] H. H. Yiu, S. C. McBain, Z. A. Lethbridge, M. R. Lees, and J. Dobson, "Preparation and characterization of polyethylenimine-coated $\mathrm{Fe}_{3} \mathrm{O}_{4}$-MCM-48 nanocomposite particles as a novel agent for magnet-assisted transfection,"
Journal of Biomedical Materials Research A, vol. 92, no. 1, pp. 386-392, 2010

[17] P. del Pino, A. Munoz-Javier, D. Vlaskou, P. Rivera Gil, C. Plank, and W. J. Parak, "Gene silencing mediated by magnetic lipospheres tagged with small interfering RNA," Nano Letters, vol. 10, no. 10, pp. 3914-3921, 2010.

[18] J. Lim, M. A. Clements, and J. Dobson, "Delivery of short interfering ribonucleic acid-complexed magnetic nanoparticles in an oscillating field occurs via caveolae-mediated endocytosis," PLoS ONE, vol. 7, no. 12, article e51350, 2012.

[19] C. Plank, O. Zelphati, and O. Mykhaylyk, "Magnetically enhanced nucleic acid delivery. Ten years of magnetofectionProgress and prospects," Advanced Drug Delivery Reviews, vol. 63, no. 14-15, pp. 1300-1331, 2011.

[20] W. Li, N. Ma, L.-L. Ong et al., "Enhanced thoracic gene delivery by magnetic nanobead-mediated vector," The Journal of Gene Medicine, vol. 10, no. 8, pp. 897-909, 2008.

[21] E. Delyagina, A. Schade, D. Scharfenberg et al., "Improved transfection in human mesenchymal stem cells: effective intracellular release of pDNA by magnetic polyplexes," Nanomedicine, 2013.

[22] A. Schade, E. Delyagina, D. Scharfenberg et al., "Innovative strategy for microRNA delivery in human mesenchymal stem cells via magnetic nanoparticles," International Journal of Molecular Sciences, vol. 14, no. 6, pp. 10710-10726, 2013.

[23] S. Bobis, D. Jarocha, and M. Majka, "Mesenchymal stem cells: characteristics and clinical applications," Folia Histochemica et Cytobiologica, vol. 44, no. 4, pp. 215-230, 2006.

[24] P. Mark, M. Kleinsorge, R. Gaebel et al., "Human mesenchymal stem cells display reduced expression of CD105 after culture in serum-free medium," Stem Cells International, vol. 2013, Article ID 698076, 8 pages, 2013.

[25] http://clinicaltrials.gov/.

[26] W. J. Rombouts and R. E. Ploemacher, "Primary murine MSC show highly efficient homing to the bone marrow but lose homing ability following culture," Leukemia, vol. 17, no. 1, pp. 160-170, 2003.

[27] R. Gaebel, D. Furlani, H. Sorg et al., "Cell origin of human mesenchymal stem cells determines a different healing performance in cardiac regeneration," PLoS ONE, vol. 6, no. 2, e15652, 2011.

[28] W. Wang, W. Li, L. Ou et al., "Polyethylenimine-mediated gene delivery into human bone marrow mesenchymal stem cells from patients," Journal of Cellular and Molecular Medicine, vol. 15, no. 9, pp. 1989-1998, 2011.

[29] Y. H. Choi, A. Kurtz, and C. Stamm, "Mesenchymal stem cells for cardiac cell therapy," Human Gene Therapy, vol. 22, no. 1, pp. 3-17, 2011.

[30] M. F. Pittenger and B. J. Martin, "Mesenchymal stem cells and their potential as cardiac therapeutics," Circulation Research, vol. 95, no. 1, pp. 9-20, 2004.

[31] A. Judge and I. MacLachlan, "Overcoming the innate immune response to small interfering RNA," Human Gene Therapy, vol. 19, no. 2, pp. 111-124, 2008.

[32] W. T. Godbey, K. K. Wu, and A. G. Mikos, "Poly(ethylenimine) and its role in gene delivery," Journal of Controlled Release, vol. 60, no. 2-3, pp. 149-160, 1999.

[33] S. Huth, J. Lausier, S. W. Gersting et al., "Insights into the mechanism of magnetofection using PEI-based magnetofectins 
for gene transfer," The Journal of Gene Medicine, vol. 6, no. 8, pp. 923-936, 2004.

[34] http://www.ozbiosciences.com/.

[35] S. N. Uddin, "Cationic lipids used in non-viral gene delivery systems," Biotechnology and Molecular Biology Review, vol. 2, pp. 58-67, 2007.

[36] Y. Zhang, W. Li, L. Ou et al., "Targeted delivery of human VEGF gene via complexes of magnetic nanoparticle-adenoviral vectors enhanced cardiac regeneration," PLOS ONE, vol. 7, no. 7, article e39490. 

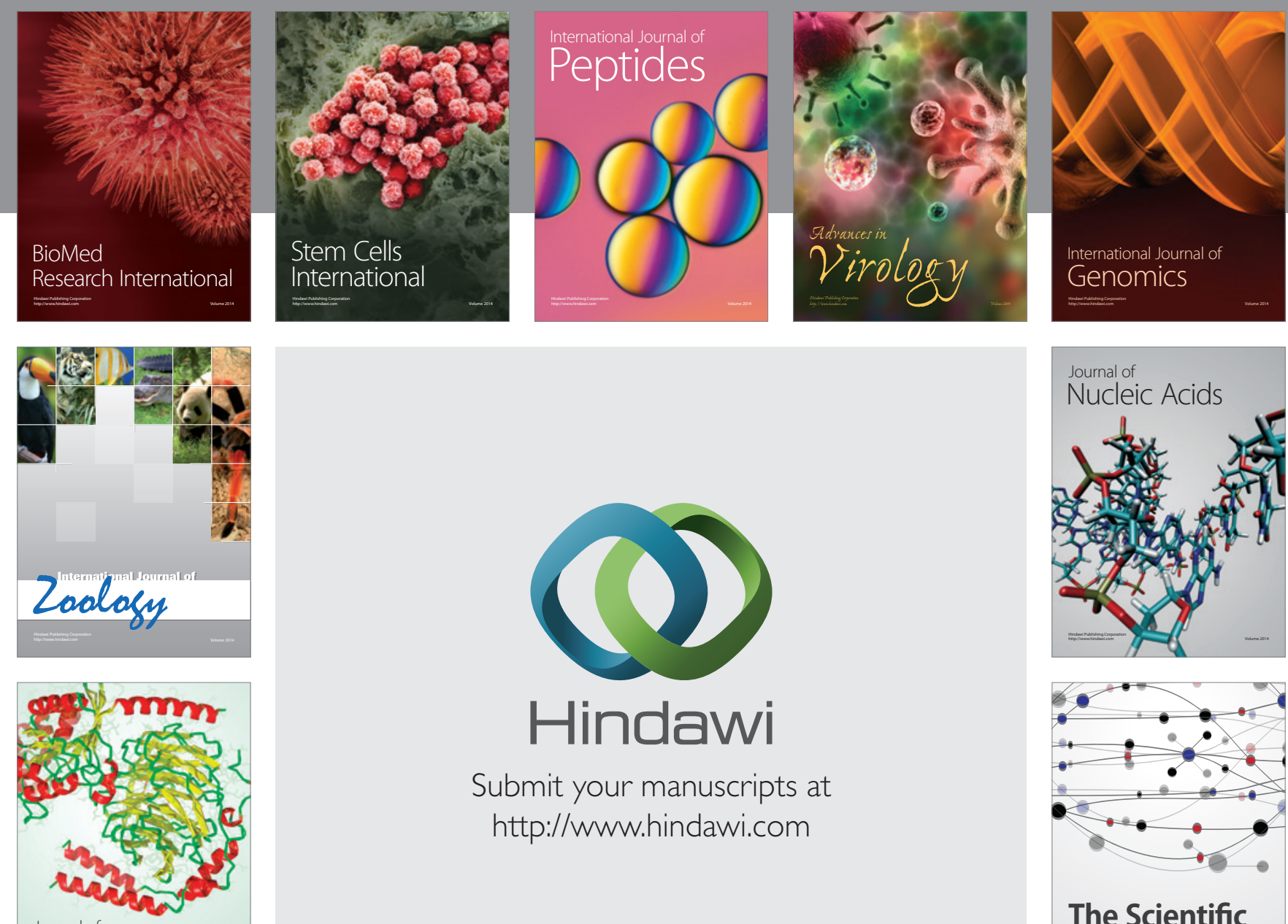

Submit your manuscripts at

http://www.hindawi.com

Journal of
Signal Transduction
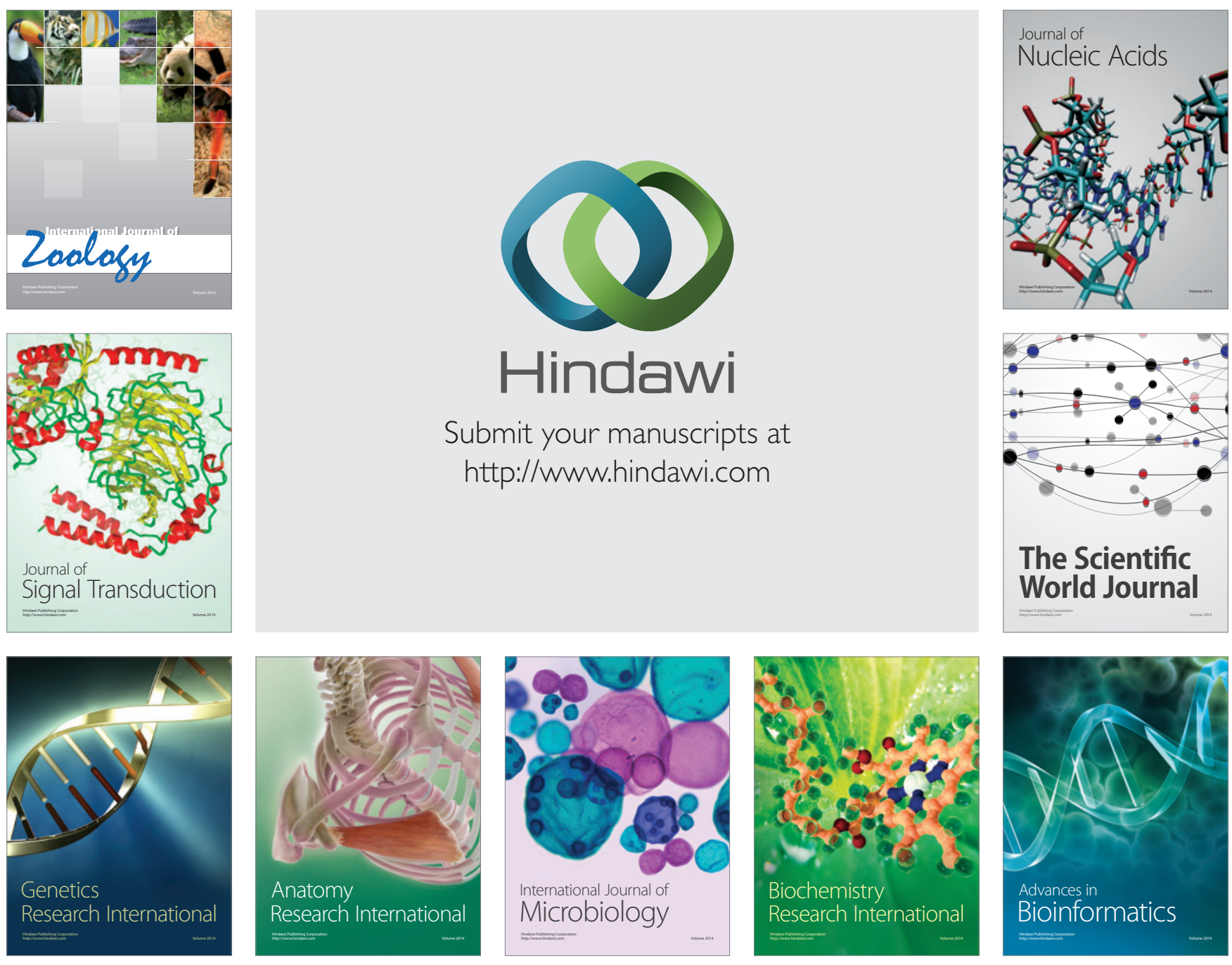

The Scientific World Journal
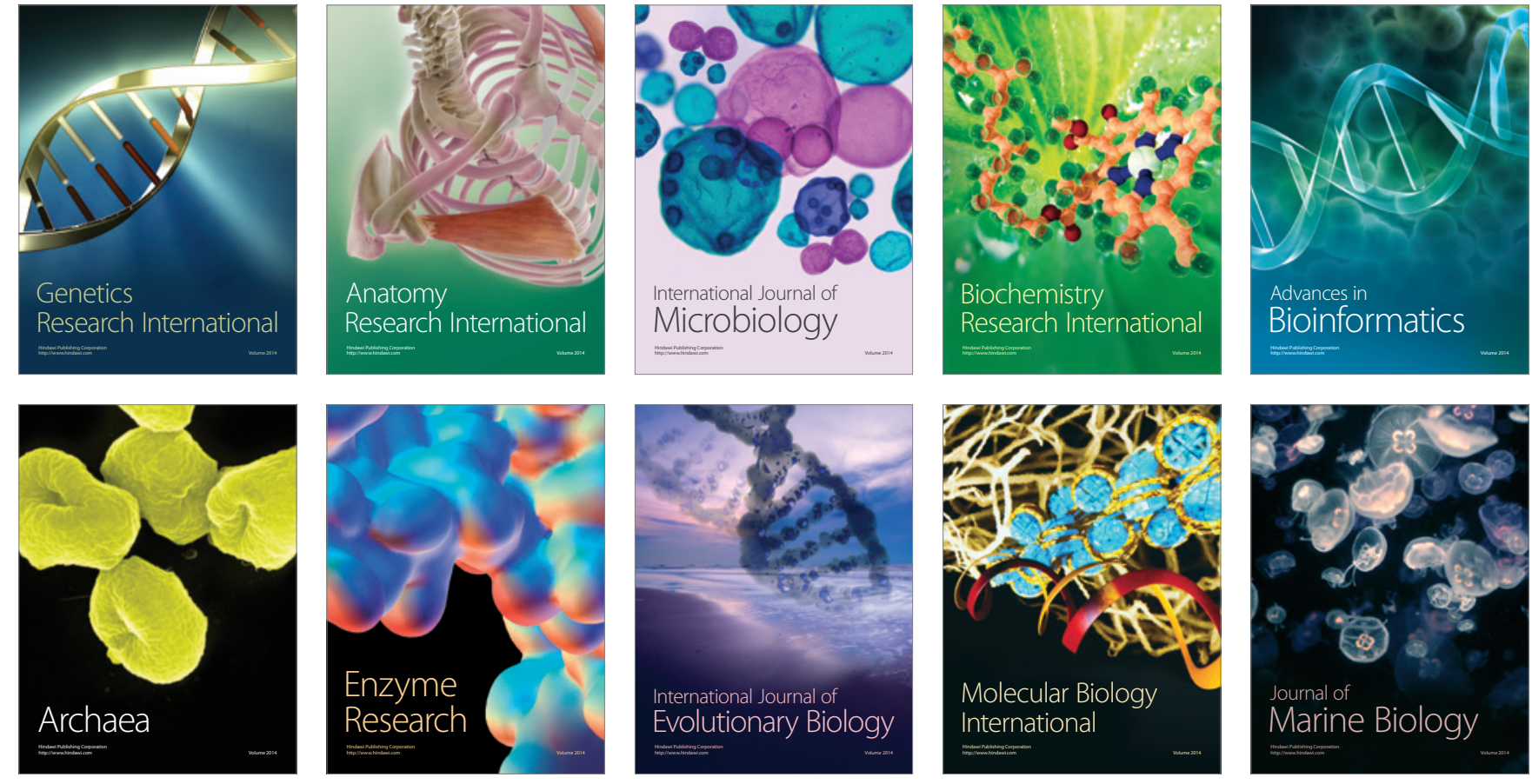